\title{
Variability in normal myocardial blood flow measurements: Physiologic, methodologic, or protocol related?
}

\author{
Timothy M. Bateman, $M D,{ }^{a, b}$ and James $A$. Case, $\mathrm{PhD}^{\mathrm{b}}$ \\ ${ }^{a}$ Saint-Lukes Cardiovascular Consultants Mid America Heart Institute, Kansas City, MO \\ ${ }^{b}$ Cardiovascular Imaging Technologies, Kansas City, MO
}

Received Sep 24, 2014; accepted Sep 25, 2014

doi: 10.1007/s12350-014-0007-1

\section{See related article, pp. 72-84}

The current issue of the Journal of Nuclear Cardiology includes an interesting investigation that studied 3 software approaches for quantitating myocardial blood flow (MBF) and MBF reserve (MBFR) in 49 subjects who met multiple criteria for having normal cardiovascular physiology: able to exercise to a high level without chest pain or ischemic end-points, no major risk factors, and normal rubidium-82 myocardial perfusion images. ${ }^{1}$ The study found that these important flow measurements in apparently normal subjects varied substantially according to age, gender, coronary territory, and software employed. The results raise legitimate questions about whether positron emission tomography (PET) derived flow quantitation is currently sufficiently mature for inclusion in clinical imaging reports that might influence subsequent patient care.

Performance of radionuclide myocardial perfusion imaging (MPI) using PET has been engendering interest and significant volume growth over the past few years. PET has been shown to offer improved image quality, interpretive certainty, and accuracy compared to both traditionally performed and attenuation-corrected single photon emission computed tomography (SPECT) MPI. ${ }^{2-7}$ PET MPI is most commonly performed with rubidium-82, a tracer that decays rapidly due to its 75 seconds half-life. Attractive attributes include

Reprint requests: Timothy M. Bateman, MD, Saint-Lukes Cardiovascular Consultants Mid America Heart Institute, Kansas City, MO; tbateman@saint-lukes.org

J Nucl Cardiol 2015;22:85-8.

1071-3581/\$34.00

Copyright (C) 2014 American Society of Nuclear Cardiology. throughput efficiency (rest/stress studies can be completed in about 40 minutes), low radiation exposure, ${ }^{8}$ peak stress regional and global ventricular function comparisons against rest parameters, ${ }^{9,10}$ and flow measurements validated against a variety of different standards. $^{11-20}$

A novel capability of PET is the ability to move beyond spatially relative image interpretation to patientcentric quantitation. In a spatially relative domain, abnormality is defined in one of 3 manners: one or two myocardial regions have less tracer uptake than a best and therefore presumed normal area; the left ventricle function deteriorates with stress (e.g., transient ischemic dilation, changes between rest and stress ejection fraction); or a non-perfusion marker such as lung uptake of Tl-201 or ischemic ECG changes alert to physiologically significant CAD not detected by perfusion defect analysis alone. PET's patient-centric quantitation advances understanding about adequacy of MBF by providing an absolute measure of both global and regional blood flow at rest, at stress, and in relative terms as a ratio between stress and rest. As such, the truly unique contribution of PET is measurement of whether the myocardium is getting the blood flow necessary to meet needs, regardless of the basis for any limitation. Identified sites of blood flow compromise include the epicardial coronary arteries (local or diffuse stenoses, endothelial dysfunction/inflammation), the microcirculation, and increased epicardial-endocardial pressure gradients. These measurements have found value in improved risk stratification in varied patient populations, and for improved recognition of multi-vessel CAD. ${ }^{21-29}$

Two important questions arise in consideration of how PET flow measurements might fit into the testing paradigm for CAD. The first is a population-based concept; on a macro-level can MBF calculations help to inform about relative coronary circulation health and improve ability to risk-stratify patients? The second 
question is whether PET flow measurements are currently robust enough to warrant inclusion in clinical reports, potentially leading to management decisions that in some cases would differ if based only on traditional perfusion and function parameters.

\section{MEASUREMENT OF MYOCARDIAL BLOOD FLOW: WHAT IT ADDS AND WHY IT IS NEEDED}

MBF studies have contributed greatly to the understanding of coronary physiology and the impact of various external influences. A recent trend among investigating centers is to measure $\mathrm{MBF}$, usually in terms of $\mathrm{MBF}$ reserve, in daily clinical practice and to track its contribution to outcomes measures. A large number of studies have shown that it adds incrementally to the detection of multivessel CAD, improves prognostication compared to perfusion defect analysis alone for normal as well as for those patients with perfusion defects, and confers a positive net reclassification index. In general, the published data suggests that a normal scan with normal MBFR connotes a very low likelihood for major adverse cardiac events, while a normal appearing scan with abnormal MBFR actually identifies a higher-risk group than one with abnormal perfusion but globally normal MBFR. Regardless of its basis, abnormal MBF carries prognostic information that is additive and incremental to perfusion defect analysis. Additional values include assurance that the vasodilator actually resulted in augmented blood flow, especially in patients who may recently have ingested a known antagonist, and in the detection of microvascular disease. Several of the above referenced studies have shown that among patients referred for PET MPI, a significant proportion have abnormal MBF reserve, commonly referenced against a two-fold increase in flow between rest and stress.

\section{ARE PET FLOW MEASUREMENTS CURRENTLY SUFFICIENTLY ROBUST FOR ALTERING MANAGEMENT?}

The pharmacologic vasodilators used for radionuclide MPI studies (dipyridamole, adenosine, regadenoson) have all been shown to augment $\mathrm{MBF}$ when administered according to their package inserts. The approved dosages and administration protocols should increase measured tracer uptake between two and four times above baseline in the presence of normal coronary physiology, depending on the specific vasodilator protocol utilized and the flow modeling algorithm employed. As such, derived flow values should at a minimum permit broad interpretive generalizations (within the confines of a particular protocol and flow modeling algorithm) useful for integration with traditional data about spatially relative perfusion and ventricular function changes between rest and stress. One example might be a stress $\mathrm{MBF}$ of 2.5 to $4 \mathrm{~mL} \cdot \mathrm{min}^{-1} \cdot \mathrm{gm}^{-1}$, and/or a MBFR reflective of at least doubling over base-line flows. Such values should imply that despite risk factors, age, and gender that the substrate-delivery system to myocardium is intact and healthy. On the opposite end of the spectrum would be no or minimal flow augmentation; until proved otherwise this should be regarded as an ineffective vasodilation often due to circulating A2a-receptor antagonists such as caffeine. Demonstrated but highly attenuated increases in flow (for example in the range of 1.3-1.6 for either MBF or MBFR) perhaps should require further investigation to determine the source of the abnormality; for example, epicardial CAD for which there are validated mechanical options that would need to be ruled out.

There are a large number of other considerations that come into play when applying such straight-forward generalizations to flow-data incorporation into decisionmaking. These include particularly the integrity of the flow measurements. Strict adherence to protocols, use of appropriate equipment, understanding the details of flow measurements, and the clinical circumstances when these are likely to be invalid, knowing how to quality control the data, and understanding the software tool in use are all essential prerequisites. Even in highly controlled research-oriented labs, flow values can be contaminated by poor blood pool identification, misalignment (sometimes precise alignment not possible) of emission and transmission data, patient motion, respiratory motion, poor counts, and scattered counts from adjacent structures, to name a few. Some patients are not likely good candidates for flow quantitation, such as those with intracardiac shunts, valvular insufficiency, very complex coronary anatomy such as after multivessel coronary bypass, and those with advanced cardiomyopathies. While flow quantitation in such patients may be feasible in theory, in practice many of the software packages that are commercially available employ modeling algorithms that are applicable to more straight-forward patients.

\section{DEPENDENCY OF NORMAL VALUES ON AGE, GENDER, VASCULAR TERRITORY, AND SOFTWARE: IMPLICATIONS}

The paper by Sunderland et al applied 3 commercially available (only one currently FDA-approved) software programs to the same data generated from " "normal', research subjects. While absolute flow estimations were significantly different between software programs, the measurements were tracked similarly. 
Importantly, the MBFR estimates that are most frequently relied on in daily practice were more homogeneous. As the authors state, "Presumably the individual software-associated $\mathrm{MBF}$ biases for both stress and rest measurements cancel one another out in the MFR ratio calculation', In the case of MBFR, there were no statistically significant differences between men and women; and mean MBFR for both were above $2.5 \times$ baseline for all 3 tested programs. MBFR values also did not significantly vary across age.

The study does, however, raise points of caution and perhaps concern. The statistically significant differences between the programs in estimating absolute rest and stress flows and MBFR imply that there are inherent differences between the programs such that when presented with the exact same data, different measurements are reported. The current investigation did not reveal the basis of these differences, but there are numerous potential sources as the programs differ as to computations of the input functions, the myocardial uptake statistics, and the assumptions about tracer rolloff at higher flow rates.

Perhaps the largest concern about the presented values lies in the reported huge variation in $\mathrm{MBF}$ and MBFR measurements in a population of normals. All of these subjects must be presumed to be able to increase MBF as they all were able to achieve high heart rates and metabolic-equivalents of exercise, certainly not conceivable if they could not increase blood flow to the myocardium. Nevertheless, 3 of the 49 demonstrated virtually no blood flow increase over rest baseline. Further, the MBFR values ranged from 1 (no change from baseline) to greater than 6 (not likely even a capability of $\mathrm{Rb}-82$ as its myocardial uptake flattens at flow levels much lower). Furthermore, approximately 8 out of the 50, dependening on the software used, had global left ventricular MBFR values below 2, a threshold for a worse prognosis in virtually all published series. Therefore, we have to be concerned that the specific findings in this study are affected by one or more of physiologic, protocol, or methodologic problems that would render the values erroneous. Possibilities for the variability range from true heterogeneity in MBF augmentation due to details of this specific population, through to artifacts related to flow modeling approaches common to all 3 softwares.

The authors of this study are to be congratulated for pushing the concept of PET-derived flow quantitation one step closer to clinical reality. Several conclusions appear reasonable. Absolute flow measurements are more problematic in terms of consistency across programs than are the relative flow estimations provided by the so-called "myocardial blood flow reserve" ratio. Providers should maintain a healthy skepticism about flow estimations, especially when these seem at the extremes of expectation. There needs to be careful attention to the details of acquisition, registration, processing, and patient-specific appropriateness for such measurements. Robust quality assurance interrogation of the data would be highly useful additions to software products. Technologists involved in the generation of these outputs and physicians charged with assessing them need to be highly trained and educated in this new sub-specialized arena and need to be very familiar with the software being used. This is essential because there are instances when MBF quantitation is at variance with the relative perfusion information; only training and close adherence to standardized protocols can help clinicians decipher such cases. At least for the timebeing, providers would be advised to predicate reporting based on local data-base driven learning rather than literature-based, which could be different depending on the details of acquisition, processing, and software employed. Software developers should be encouraged to adopt a common approach to quantitating and qualitycontrolling these important measurements such that imagers and care-givers can have greater confidence in the values. They should also be encouraged to understand the cause of different flow values between different programs. Professional medical societies should insist on common standards through guidelines development.

Finally, the study appears to indicate that it is still too early in the evolution of this extremely intriguing addition to radionuclide MPI to envision cross-vendor multicenter studies, so badly needed in order to drive guidelines for incorporation of flow quantitation into decision-making.

\section{References}

1. Sunderland JJ, Pan X-B, Declerck J, Menda Y. Dependency of cardiac rubidium-82 imaging quantitative measures on age, gender, vascular territory, and software in a cardiovascular normal population. J Nucl Cardiol 2014. doi:10.1007/s12350-014-9920-6.

2. Bateman TM, Heller GV, McGhie AI, Friedman JD, Case JA, Bryngelson JR, et al. Diagnostic accuracy of rest/stress ECG-gated Rb-82 myocardial perfusion PET: Comparison with ECG-gated Tc- 99m sestamibi SPECT. J Nucl Cardiol 2006;13:24-33.

3. Yoshinaga K, Chow BJW, Williams KA, Chen L, deKemp RA, Garrard L, et al. What is the prognostic value of myocardial perfusion imaging using rubidium-82 positron emission tomography? J Am Coll Cardiol 2006;48:1029-39.

4. Kajander S, Joutsiniemi E, Saraste M, Pietilä M, Ukkonen H, Saraste A, et al. Cardiac positron emission tomography/computed tomography imaging accurately detects anatomically and functionally significant coronary artery disease. Circulation 2010;122:603-13

5. McArdle BA, Dowsley TF, de Kemp RA, Wells GA, Beanlands RS. Does rubidium-82 PET have superior accuracy to SPECT 
perfusion imaging for the diagnosis of obstructive coronary disease: A systematic review and meta-analysis. J Am Coll Cardiol 2012;60:1828-37.

6. Parker MW, Iskandar A, Limone B, Perugini A, Kim H, Jones C, et al. Diagnostic accuracy of cardiac positron emission tomography versus single photon emission computed tomography for coronary artery disease: A bivariate meta-analysis. Circ Cardiovasc Imaging 2012;5:700-7.

7. Sampson UK, Dorbala S, Limaye A, Kwong R, DiCarli M. Diagnostic accuracy of rubidium-82 myocardial perfusion imaging with hybrid positron emission tomography/computed tomography in the detection of coronary artery disease. J Am Coll Cardiol 2007;49:1052-8

8. Cerqueira MD, Allman KC, Ficaro EP, Hansen CL, Nichols KJ, Thompson RC, et al. Recommendations for reducing radiation exposure in myocardial perfusion imaging: ASNC Information Statement. J Nucl Cardiol 2010;17:709-18.

9. Dorbala S, Hachamovitch R, Curillova Z, Thomas D, Vangala D, Kwong RY, et al. Incremental prognostic value of gated Rb-82 positron emission tomography myocardial perfusion imaging over clinical variables and rest LVEF. J Am Coll Cardiol Imaging 2009;2:846-54.

10. Lertsburapa K, Ahlberg AW, Bateman TM, Katten D, Volker L, Cullom SJ, et al. Independent and incremental prognostic value of left ventricular ejection fraction determined by stress gated rubidium 82 PET imaging in patients with known or suspected coronary artery disease. J Nucl Cardiol 2008;15:745-53.

11. Lortie M, Beanlands RSB, Yoshinaga K, Klein R, Dasilva JN, Dekemp RA. Quantification of myocardial blood flow with 82Rb dynamic PET imaging. Eur $\mathrm{J}$ Nucl Med Mol Imaging 2007;34:1765-74

12. El Fakhri G, Kardan A, Sitek A, Dorbala S, Abi-Hatem N, Lahoud $\mathrm{Y}$, et al. Reproducibility and accuracy of quantitative myocardial blood flow assessment with 82Rb PET: Comparison with $13 \mathrm{~N}$ ammonia PET. J Nucl Med 2009;50:1062-71.

13. Manabe O, Yoshinaga K, Katoh C, Naya M, de Kemp RA, Tamaki N. Repeatability of rest and hyperemic myocardial blood flow measurements with Rb82 dynamic PET. J Nucl Med 2009;50:6871.

14. Klein R, Renaud JM, Ziadi MC, Thorn SL, Adler A, Beanlands RS, de Kemp RA. Intra- and inter-operator repeatability of myocardial blood flow and myocardial flow reserve measurements using rubidium- 82 pet and a highly automated analysis program. $\mathrm{J}$ Nucl Cardiol 2010;17:600-16

15. Yoshinaga K, Katoh C, Manabe O, Klein R, Naya M, Sakakibara $\mathrm{M}$, et al. Incremental diagnostic value of regional myocardial blood flow quantification over relative perfusion imaging with generator-produced rubidium-82 PET. Circ J 2011;75:2628-34.

16. Efseaff M, Klein R, Ziadi MC, Beanlands RS, de Kemp RA. Short-term repeatability of resting myocardial blood flow measurements using rubidium-82 PET imaging. J Nucl Cardiol 2012;19:997-1006.

17. Prior JO, Allenbach G, Valenta I, Kosinski M, Burger C, Verdun $\mathrm{FR}$, et al. Quantification of myocardial blood flow with $82 \mathrm{Rb}$ positron emission tomography: Clinical validation with $15 \mathrm{O}-$ water. Eur J Nucl Med Mol Imaging 2012;39:1037-47.

18. Dekemp RA, Declerck J, Klein R, Pan XB, Nakazato R, Tonge C, et al. Multisoftware reproducibility study of stress and rest myocardial blood flow assessed with 3D dynamic PET/CT and a 1tissue-compartment model of $82 \mathrm{Rb}$ kinetics. J Nucl Med 2013;54:571-7.

19. Renaud JM, Da Silva JN, Beanlands RSB, de Kemp RA. Characterizing the normal range of myocardial blood flow with Rb82 and N13-ammonia PET imaging. J Nucl Cardiol 2013;20:578-91.

20. Tahari AK, Lee A, Rajaram M, Fukushima K, Lodge MA, Lee $\mathrm{BC}$, et al. Absolute myocardial flow quantification with (82)Rb PET/CT: Comparison of different software packages and methods. Eur J Nucl Med Mol Imaging 2014;41:126-35.

21. Herzog BA, Husmann L, Valenta I, Gaemperli O, Siegrist PT, Tay FM, et al. Long-term prognostic value of N13-ammonia myocardial perfusion positron emission tomography: Added value of coronary flow reserve. J Am Coll Cardiol 2009;54:150-6.

22. Ziadi MC, de Kemp RA, Williams KA, Guo A, Chow BJ, Renaud $\mathrm{JM}$, et al. Impaired myocardial flow reserve on rubidium- 82 positron emission tomography imaging predicts adverse outcomes in patients assessed for myocardial ischemia. J Am Coll Cardiol 2011;58:740-8.

23. Murthy VL, Naya M, Foster CR, Hainer J, Gaber M, Di Carli G, et al. Improved cardiac risk assessment with non-invasive measures of coronary flow reserve. Circulation 2011;124:2215-24.

24. Ziadi MC, Chow BJ, Renaud JM, Dekemp RA, Williams K, Guo A, et al. Does quantification of myocardial flow reserve using rubidium-82 positron emission tomography facilitate detection of multivessel coronary artery disease? J Nucl Cardiol 2012;19:67080 .

25. Murthy VL, Naya M, Foster CR, Gaber M, Hainer J, Klein J, et al. Association between coronary vascular dysfunction and cardiac mortality in patients with and without diabetes mellitus. Circulation 2012;126:1858-68.

26. Dorbala S, Di Carli MF, Beanlands RS, Merhige ME, Williams BA, Veledar E, et al. Prognostic value of stress myocardial perfusion positron emission tomography: Results from a multicenter observational registry. J Am Coll Cardiol 2013;61:176-84.

27. Farhad H, Dunet V, Bachelard K, Allenbach G, Kaufmann PA, Prior JO. Added prognostic value of myocardial blood flow quantitation in rubidium-82 positron emission tomography imaging. Eur Heart J Cardiovasc Imaging 2013;14:1203-10.

28. Naya M, Murthy VL, Taqueti VR, Foster CR, Klein J, Garber M, et al. Preserved coronary flow reserve effectively excludes highrisk coronary artery disease on angiography. J Nucl Med 2014;55:248-55.

29. Chow BJW, Dorbala S, DiCarli MF, Merhige ME, Williams BA, Veledar E, et al. Prognostic value of PET myocardial perfusion imaging in obese patients. J Am Coll Cardiol Imaging 2014;7:27887. 